\title{
Thrombektomie beim akuten Schlaganfall - Schritt für Schritt
}

\author{
Joachim Berkefeld, Fee Keil
}

\begin{abstract}
Die kathetergestützte Thrombektomie beim akuten Schlaganfall hat sich zu einer Evidenz-basierten Standardbehandlung entwickelt. Die Durchführung erfordert ein standardisiertes Vorgehen, das für drei Standardtechniken beschrieben wird, um das Verständnis für die Abläufe und technische Durchführung bei Neurologen zu erhöhen.
\end{abstract}

\section{Grundlagen}

Die interventionelle Schlaganfallbehandlung mit Thrombektomie ist bei klinisch schwer betroffenen Patienten mit embolischen Verschlüssen großer intrakranieller Arterien vielfach als Methode der Wahl anzusehen. Randomisierte Studien haben die Überlegenheit der kathetergestützten Therapie gegenüber einer i.v.Thrombolyse oder sonstigen medikamentösen Behandlung in Zeitfenstern von bis zu 24 Stunden nach Symptombeginn nachgewiesen [1,2]. Heute aktuelle und durch Studien gesicherte Indikationen und Techniken werden in komprimierter Form und anhand von Zeichnungen und Bildbeispielen dargestellt.

Der Schlaganfall ist ein zeitkritischer Notfall, bei dem alle Schritte der Diagnostik und Therapie so schnell wie möglich und ohne Verzögerung durchgeführt werden müssen. Entsprechende Team-Trainings und Ablaufschemata sowie diagnostische und interventionelle Standards erleichtern dies sehr.

\section{Wann ist eine Thrombektomie indiziert?}

\section{Diagnostik}

Die Indikationsstellung zur Thrombektomie ist bei klinisch schwer betroffenen Schlaganfallpatienten unverzüglich zu prüfen. Der Rettungsdienst sollte bereits Patienten mit einer ausgeprägten Hemiparese oder Sprachstörung identifizieren und unverzüglich in eine geeignete Klinik bringen. Der Neurologe legt dort anhand des NIHSS den Schwergrad der Ausfälle fest. Eine Thrombektomie ist klinisch bei einem relevanten und behindernden neurologischen Defizit indiziert, wenn ein embolisch bedingter Verschluss einer großen intrakraniellen Arterie vorliegt.
Nach einer kurzen Sichtung des Patienten ist unverzüglich eine bildgebende Diagnostik mit Darstellung des Hirnparenchyms und der hirnversorgenden Gefäße einzuleiten, um dies festzustellen [3].

Als breit verfügbare und schnelle Standardmethode hat sich die native Schädel-CT in Kombination mit einer CT-Angiographie vom Aortenbogen bis nach intrakraniell bewährt.

Mit der CT-Diagnostik sind folgende diagnostische Aussagen zu treffen:

- Ausschluss einer Blutung

- Infarktausmaß: Infarktfrühzeichen in Form von Dichteminderungen, Bewertung nach dem ASPECTS ([4], > Abb. 1)

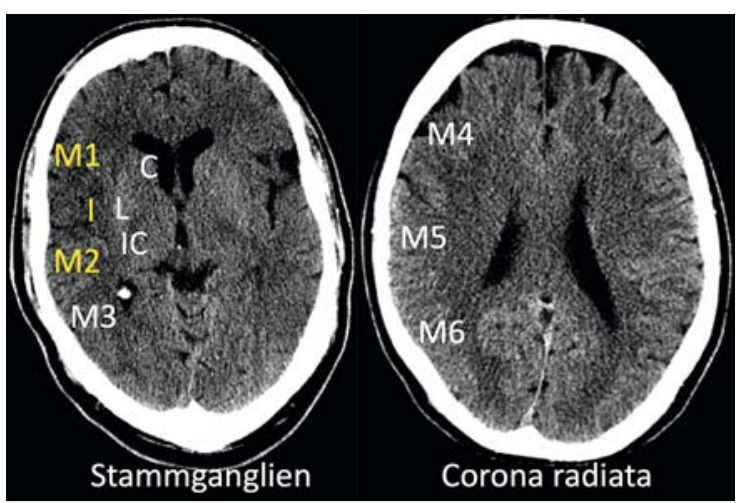

- Abb. 1 Festlegung der Infarktausdehnung mit Hilfe des ASPECTS am Beispiel eines Patienten mit einem Rechtshemisphärensyndrom. Einteilung des Mediaterritoriums in zwei repräsentativen CT-Schichten in 10 Referenzregionen: I = Insel, C = Caudatuskopf, L = Linsenkern, IC = innere Kapsel, M1-M6= kortikale Areale im Mediaterritorium. Suche nach dichtegeminderten Arealen mit enger Fenstereinstellung; hier: M1, I und M2 (gelbe Buchstaben). Der ASPECTS gibt die Zahl der intakten Areale ohne Dichteminderung an: in unserem Fall liegt der ASPECTS bei 7 Punkten. 
- Lokalisation und Länge des Gefäßverschlusses

- Einschätzung der Kollateralversorgung

Fallbeispiel 1a zeigt die diagnostischen Schritte der CT- und CTA-Beurteilung ( $\mathbf{A b b}$. 2).

Noch am Gerät legen Neurologen und Neuroradiologen das weitere Vorgehen fest:

Wenn keine Kontraindikationen bestehen, wird noch im CT-Raum eine intravenöse Thrombolysebehandlung begonnen.

Eine klare Thrombektomie-Indikation besteht, wenn folgende Punkte erfüllt sind:

- NIHSS $\geq 6$ Punkte

- Proximaler Gefäßverschluss:

- intrakranielle ACI, Carotis-T, M1-Segment, dominanter M2-Ast im vorderen Hirnkreislauf

- A. basilaris im hinteren Hirnkreislauf

- Tandemläsionen: Kombination von Carotisstenose/Verschluss extrakraniell + nachgeschaltetem intrakraniellem Verschluss durch eine arterio-arterielle Embolie.

- Zeitfenster seit Symptombeginn von bis zu 6 Stunden, in ausgewählten Fällen mit begrenztem Infarktkern auch bis zu 24 Stunden.

- Rettbares Hirnparenchym mit begrenzten Infarktfrühzeichen (ASPECTS > 5).
In fortgeschrittenen Zeitfenstern oder bei unsicherem Infarktausmaß in der CT kann eine MRT-Diagnostik sinnvoll sein, um festzustellen, ob über den im Diffusionsbild leicht erkennbaren Infarktkern hinaus noch gefährdetes Hirnparenchym mit in der MR-Perfusionsmessung kritischer Minderperfusion vorliegt. Alternativ kann ein sog. Mismatch zwischen begrenztem Infarktkern und minderperfundiertem, noch rettbarem Hirnparenchym in der Umgebung auch durch eine CTPerfusionsmessung festgestellt werden. Hierbei wird der Infarktkern durch ein kritisch vermindertes Blutvolumen oder eine ausgeprägte Verzögerung der Kontrastmittel-Anflutung festgestellt.

Für folgende Indikationen besteht noch weiterer Studienbedarf. Nach bisherigen Daten können diese Patienten im gemeinsam mit den Neurologen zu entscheidenden Einzelfall von einer Thrombektomie profitieren:

- M2-Astverschlüsse jenseits der Mediabifurkation bei schwer betroffenen Patienten.

- großer Infarktkern ASPECTS 3 - 5

- klinisch leicht betroffene Patienten mit NIHSS <6 und proximalem Gefäßverschluss

\section{Logistik der Thrombektomie}

Nach der Indikationsstellung sollte der Patient unverzüglich der Thrombektomie zugeführt werden. Wenn die Erstversorgung in einem Thrombektomie-Zentrum
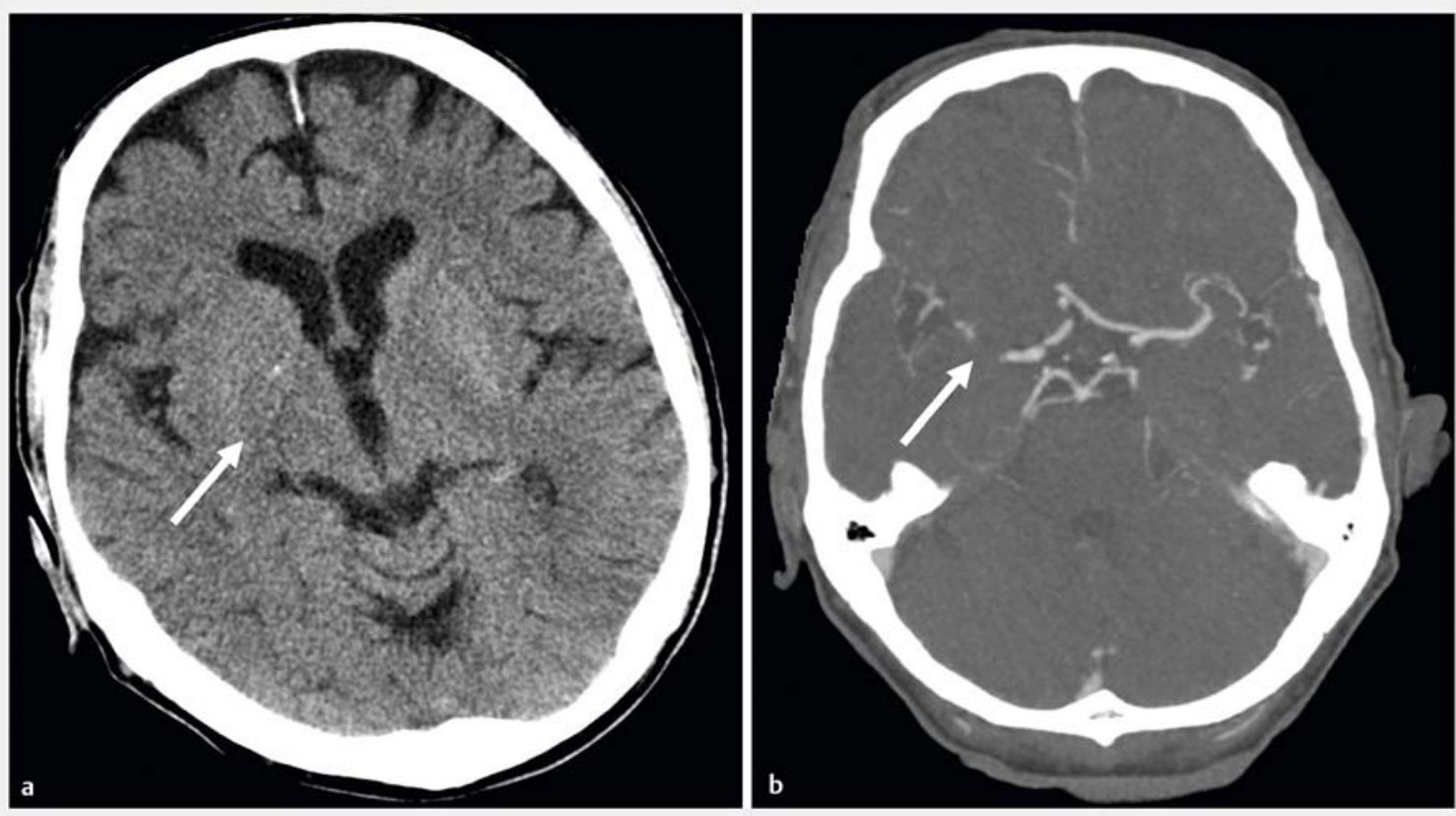

- Abb. 2 Fallbeispiel 1a CCT und CT-Angiographie eines 75 jährigen Patienten mit plötzlich vor 2 Std. aufgetretener Hemiplegie links und leichter Bewusstseinstrübung. a Die native CT zeigt eine geringe Dichteminderung im Stammgangliengebiet re. (Pfeil) bei sonst unauffälliger Darstellung des Hirnparenchyms (ASPECTS=9). b CT-angiographischer Nachweis eines rechtsseitigen M1-Verschlusses (Pfeil). 
erfolgt, werden Anästhesie und Interventionsteam informiert, sobald klar ist, dass ein Thrombektomie-Kandidat im Hause ist. Die Abläufe erfolgen am besten nach einem standardisierten Schema, um zeitliche Verzögerungen zu vermeiden [5]. Bei Verlegungen aus Kliniken ohne Thrombektomie-Möglichkeit ist der Transport in ein Thrombektomie-Zentrum sofort nach der CT-Diagnostik zu organisieren. Vorteilhaft ist es beispielsweise, wenn der Rettungsdienst bis zum Abschluss der CT wartet und gleich danach den Weitertransport übernimmt. Aus der Qualitätssicherung bekannt gewordene Zeiten von über einer Stunde von der CT bis zum Beginn der Verlegung sind nicht akzeptabel. Sekundär in ein Thrombektomie-Zentrum verlegte Patienten sollten beim Eintreffen sofort in die Angiographie gebracht werden. Bei sehr langen Zeiten von mehr als zwei Stunden zwischen der initialen Bildgebung und der Ankunft im Thrombektomie-Zentrum ist eine Aktualisierung der Bildgebung z.B. mit DWI im MRT zu diskutieren, um frustrane Behandlungen von Patienten mit zwischenzeitlich aufgetretenen ausgedehnten Infarkten zu vermeiden.

\section{Vorbereitung des Patienten}

Für die Durchführung einer Thrombektomie ist eine Intubationsnarkose (ITN) oder Sedierung erforderlich. Beide Möglichkeiten sind in neueren als mindestens gleichwertig mit Vorteilen für die ITN betrachten [6]. Eine Thrombektomie in ITN ist bei unruhigen nicht kooperationsfähigen Patienten, bei Interventionalisten mit geringerer Erfahrung, bei komplexen Eingriffen wie der Behandlung von Tandemläsionen und bei peripheren M2-Astverschlüssen zu bevorzugen. In Absprache mit der Anästhesie sollten die Zeiten für die Einleitung und das Legen von Zugängen so kurz wie möglich gehalten werden. In vielen Fällen ist z. B. keine arterielle Blutdruckmessung erforderlich.

Es ist erforderlich, Standardmaterialien für die Thrombektomie festzulegen und griffbereit im Angiographie-Raum vorzuhalten. Die Vorbereitung des Instrumententischs und von drei Dauerspülungen mit heparinisierter Kochsalzlösung (1000l. E./ml) sollte unverzüglich nach Indikationsstellung erfolgen. Eine zusätzliche antithrombotische Behandlung während des Eingriffs ist in der Regel nicht erforderlich, um das Blutungsrisiko nicht zu erhöhen. Die prophylaktische Gabe von Kalziumantagonisten wie Nimodipin in der Spüllösung ist ebenfalls routinemäßig nicht indiziert und allenfalls bei jungen, sehr Spasmus-empfindlichen Patienten mit einer Dosis von $2 \mathrm{mg} / \mathrm{l}$ zu diskutieren.

Die Planung des Zugangs und des Eingriffs sowie die Auswahl der Materialien erfolgt nach Analyse der CTAngiographie, so dass in der Regel eine Sondierung des zu behandelnden Gefäßes ausreicht und eine diagnostische 4-Gefäß-Angiographie entbehrlich ist.

\section{Zugang für die Thrombektomie}

Nach Punktion der A. femoralis in der Leiste wird zunächst ein Diagnostikkatheter eingebracht und das zu behandelnde Gefäß sondiert. Die initiale Angiographie soll folgende Informationen liefern:

- Bestätigung der Diagnose und der Lokalisation eines intrakraniellen Gefäßverschlusses

- Lyseerfolg

- Nachweis von eventuellen Tandemläsionen mit vorgeschalteter Stenose

Als Zugang für die Thrombektomie im wird eine großlumige lange Schleuse mit 6-F-Innenlumen oder ein Ballonführungskatheter benötigt. Die Verwendung von Ballonführungskathetern, die ein Bergen von Thromben unter temporärem Verschluss der ACl erlauben hat sich im Vergleich zu langen Schleusen oder distal Access-Kathetern als vorteilhaft für das Rekanalisationsergebnis erwiesen [7].

Diese Zugangskatheter werden entweder im Austausch gegen den Diagnostikkatheter über einen in die A. carotis externa oder in die A. subclavia einbrachten langen Wechselführungsdraht eingewechselt. Alternativ und bei schwierigen Zugängen hat sich auch eine Tele-

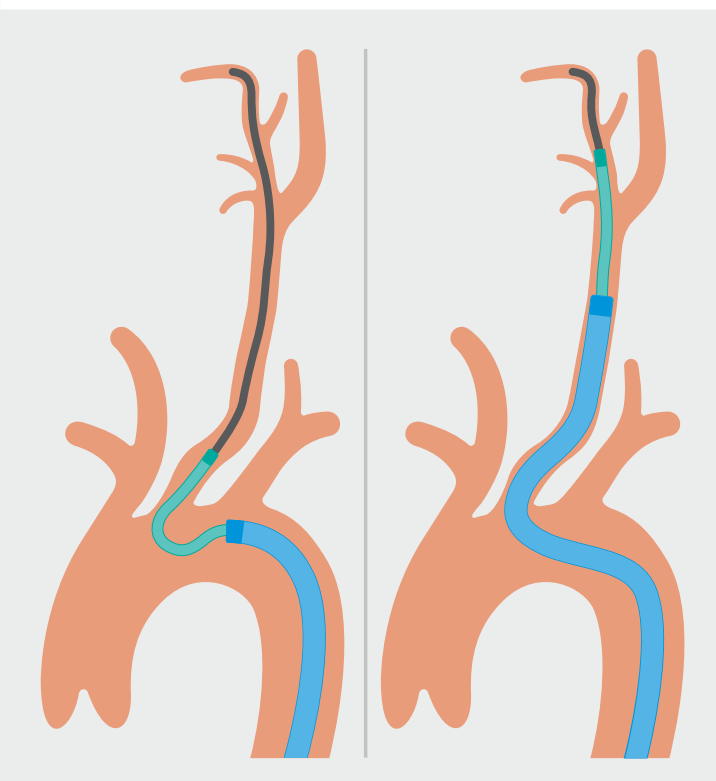

Sondierung der ACE mit überlangem Diagnostikkatheter und FK mit Führungsdraht in der ACE Vorschieben des FK in die ACC

Abb. 3 Schematische Darstellung der Teleskop-Technik mit Einbringen einer langen Schleuse oder eines Ballonführungskatheters in die zu behandelnde A. carotis. Bei einfachem Zugang kann der Zugangskatheter auch über einen in die A. carotis externa eingebrachten Wechselführungsdraht eingewechselt werden. 
skop-Technik ( $\mathbf{A}$ bb. 3) mit Einbringen des Zugangssystems über einen überlangen Diagnostikkatheter bewährt.

\section{Thrombektomie-Techniken}

Der Rückzug mit einem Stent-Retriever oder die direkte Aspiration von Thromben mit einem großlumigen Aspirationskatheter (A Direct Aspiration First Pass Technique, ADAPT) haben sich als Standardtechniken mit in Studien nachgewiesener Effektivität etabliert. Bezüglich der Rekanalisationsergebnisse und der klinischen Behandlungserfolge gibt es keine signifikanten Unterschiede [8]. In jedem Thrombektomie-Zentrum ist eine Entscheidung über die Standard-Technik der ersten Wahl zu treffen. Aus der Vielzahl von Stent-Retriever-Modellen und Kathetersystemen ist ebenfalls eine Auswahl zu treffen, um Fehler oder suboptimale Ergebnisse durch mangelnde Übung zu vermeiden.

Anhand der Illustrationen und Fallbeispiele werden die verschiedenen Schritte der Thrombektomie in unterschiedlichen Szenarien nach Einbringen des Zugangssystems durchgespielt.

\section{Standardtechnik A: Stent-Retriever + Ballonführungskatheter} siehe $>$ Abb. 4, Fallbeispiel 1b ( Abb. 5), \Video 1)

Der Stent-Retriever muss an die Thrombuslänge und an den Durchmesser des zu behandelnden Gefäßes angepasst und ein entsprechend passender Mikrokatheter zum Einführen ausgewählt werden.

Schritt 1: Über den Führungskatheter wird ein für die Platzierung des Stent-Retrievers geeigneter Mikrokatheter mit einem Mikroführungsdraht in Richtung auf das verschlossene Gefäß navigiert. Der Thrombus wird mit dem Mikroführungsdraht passiert und die Drahtspitze distal des Thrombus in einen größeren Ast zweiter Ordnung bewegt. Es ist darauf zu achten, dass der Mikrodraht mit seiner vorgebogenen Spitze nicht in dünne perforierende Arterien abweicht und diese verletzt.

Schritt 2: An der am häufigsten betroffenen Mediabifurkation ist es vorteilhaft, den Mikrodraht und den nachgeführten Mikrokatheter in der meist kaliberkräftigeren unteren M2-Astgruppe (Tr. inferior) zu platzieren. Diese biegt hinter der Bifurkation nach hinten in Richtung auf die Fissura Sylvii um, was im Seitbild gut zu erkennen ist. Die frontale Astgruppe (Tr. superior) ist in der Regel kaliberschwächer, verläuft nach vorn oben und verzweigt sich bald in noch dünnere Arterien. Es ist weniger wahrscheinlich, dass der Hauptanteil des Thrombus in diesem dünneren Ast steckenbleibt. Dem entsprechend gibt es Hinweise darauf, dass die Erfolgs- rate bei Thrombektomien über den Tr. inferior höher ist [9].

Die Position des Mikrokatheters in einem größeren Gefäß sollte durch eine vorsichtige Injektion von Kontrastmittel verifiziert werden, um eine versehentliche Sondierung eines perforierenden Astes auszuschließen.

Schritt 3: Der in einer Hülle befindliche Stent-Retriever wird in den mit der Dauerspülung verbundenen $\mathrm{Y}$ Adapter am Mikrokatheter eingeführt und gespült, bis Kochsalzlösung austritt. Anschließend wird die Hülle an den Hub des Mikrokatheters angedockt und der Stent-Retriever vorgeschoben bis die Markierungen der Spitze distal des Thrombus erkennbar sind. Es ist darauf zu achten, dass der zwischen proximalem und distalem Marker befindliche Stent-Retriever den Thrombus überdeckt. Die Freisetzung erfolgt durch langsamen Rückzug des Mikrokatheters während der Stent-Retriever in Position gehalten wird und sich dann distal des Thrombus öffnet.

Schritt 4: Wenn das distale Ende Wandkontakt hat, wird der Stent durch weiteren Rückzug des Mikrokatheters freigesetzt. Die meisten Hersteller empfehlen nach der Freisetzung eine Wartezeit von beispielsweise 5 Minuten, damit sich die Maschen des Stents durch die Expansionskräfte in die Thrombusoberfläche hineindrücken können. Bei im Röntgen sichtbaren Stent-Retrievern kann man die dadurch bedingte Expansion direkt beobachten. Am Ende der Wartezeit wird der Ballon des Führungskatheters inflatiert.

Eine Vakuumspritze wird an den Y-Konnektor des Führungskatheters angeschlossen und bei geschlossenem Hahn wird durch Anziehen der Spritze das Vakuum erzeugt, das durch Umlegen des Hahns wirksam wird. Alternativ kann auch eine Vakuumpumpe verwendet werden.

Schritt 5: Der Stent-Retriever wird langsam bis in den Y-Konnektor hinein zurückgezogen. Dieser wird dann am besten entfernt und man beobachtet, ob der Führungskatheter rückläufig ist. Ist dies nicht der Fall, wird nochmals aspiriert. Der Ballon wird deflatiert und bei frei rückläufigem Führungskatheter erfolgt die Kontrollangiographie.

Schritt 6: Angiographisch ist zu überprüfen, ob ein ausreichendes Rekanalisationsergebnis, mindestens TICI IIb erreicht wurde. Bei persistierendem Verschluss oder Embolisation eines größeren Thrombusanteils in einen abhängigen Seitenast ist das ThrombektomieManöver zu wiederholen. Insbesondere unerfahrene Operateure seien davor gewarnt, Thrombusfragmente aus weit distal der Mediabifurkation gelegenen M2 oder M3-Ästen zu entfernen. Hier drohen Blutungs- 

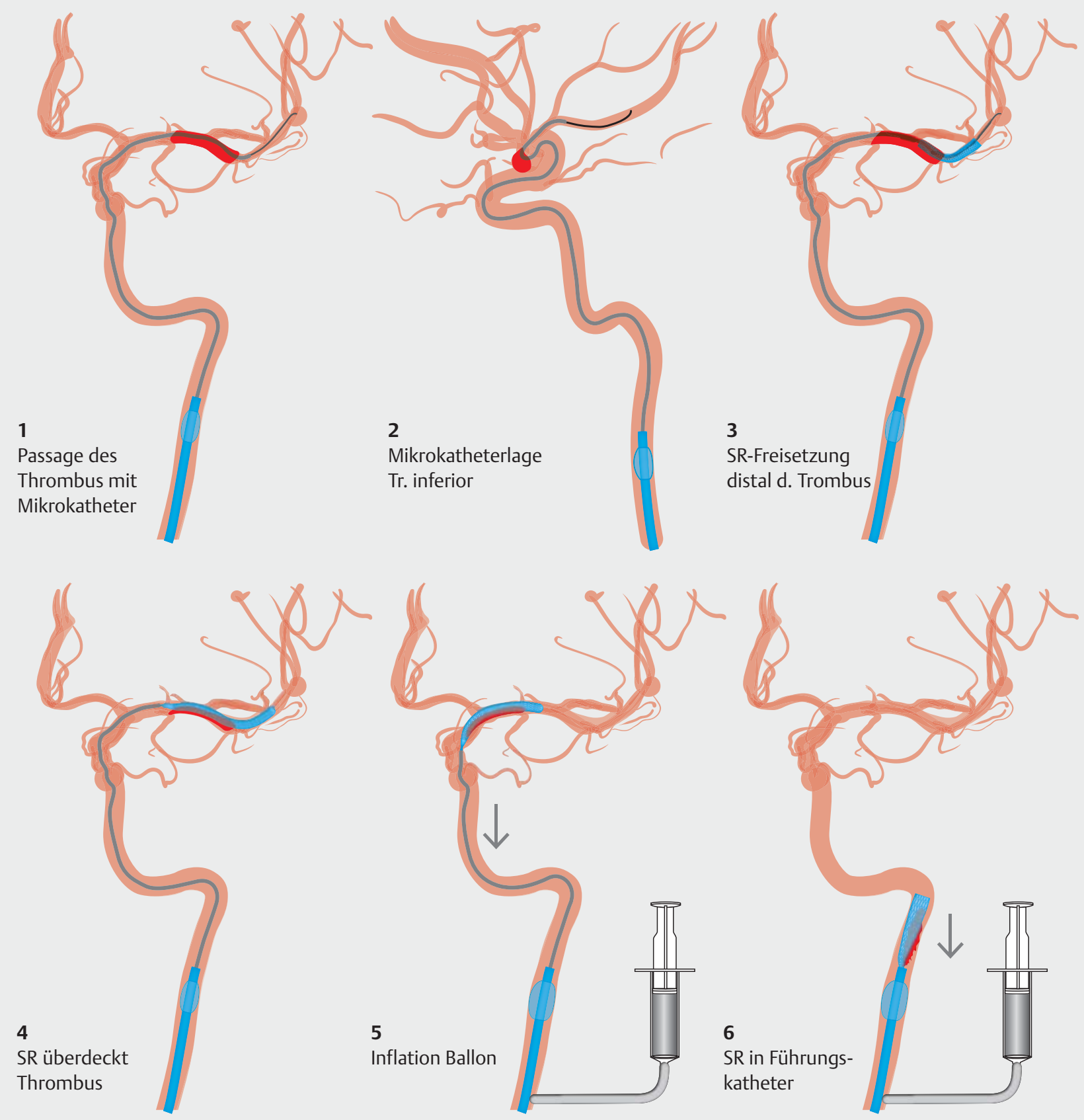

- Abb.4 Standardisiertes Vorgehen bei der Thrombektomie mit Stent-Retriever und Ballonführungskatheter. SR=Stent-Retriever.

komplikationen durch Dissektionen oder Abriss von Perforatoren. Nach drei erfolglosen Stent-RetrieverManövern werden die Erfolgsaussichten einer Thrombektomie zunehmend geringer. Dies liegt entweder an rigidem Thrombusmaterial, dass mit den Maschen des
Stent-Retrievers nicht ausreichend interagiert oder daran, dass es sich nicht um einen embolischen, sondern um einen atherothrombotischen Verschluss auf dem Boden einer Stenose handelt, die ggf. mit Ballonangioplastie oder akutem Stenting zu behandeln ist [10]. 


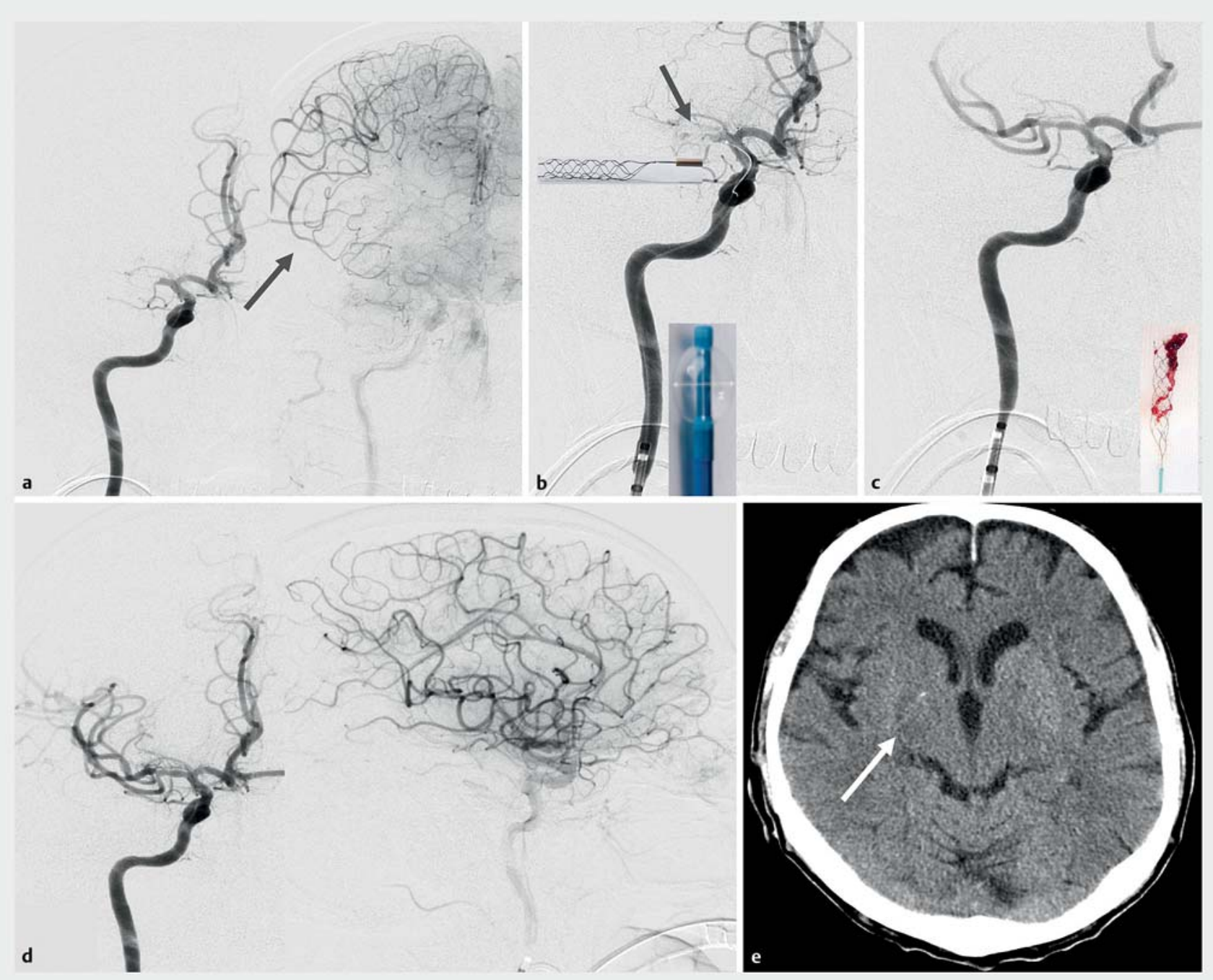

Abb.5 Fallbeispiel 1b Thrombektomie mit Stent-Retriever und Ballonführungskatheter (gleicher Patient wie Fallbeispiel 1a). a Angiographische Bestätigung der Diagnose eines M1-Verschlusses re. mit guter Kollateralversorgung aus Ästen der rechten A. cerebri anterior (Pfeil). b Nach Einwechseln eins 8 F-Ballonführungskatheters wurde ein Stent-Retriever über dem Thrombus freigesetzt. Nur geringe Kontrastierung des Gefäßlumens nach Stent-Entfaltung (Pfeil). c Wiedereröffnung des verschlossenen M1-Segments nach einmaligem Rückzug des Stent-Retrievers unter Aspiration und Ballonokklusion der ACI. d Vollständige Rekanalisation des Mediaterritorius TICI III. e CCT nach 24h: Unverändert kleiner Infarkt im Stammgangliengebiet rechts (Pfeil). Im Verlauf gute Rückbildung der klinischen Symptome.

\section{VIDEO 1}
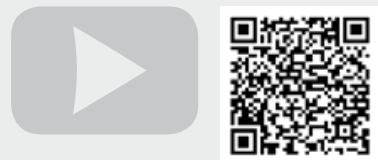

-Video 1 Präinterventionelle DSA, Freisetzung und Rückzug eines Stent-Retrievers am Beispiel eines M1-Verschlusses rechts. Erfolgreiche Rekanalisation in der postinterventionellen DSA.

https://doi.org/

\section{Standardtechnik B: Direkte Thrombusaspiration}

10.1055/a-0970-2871 


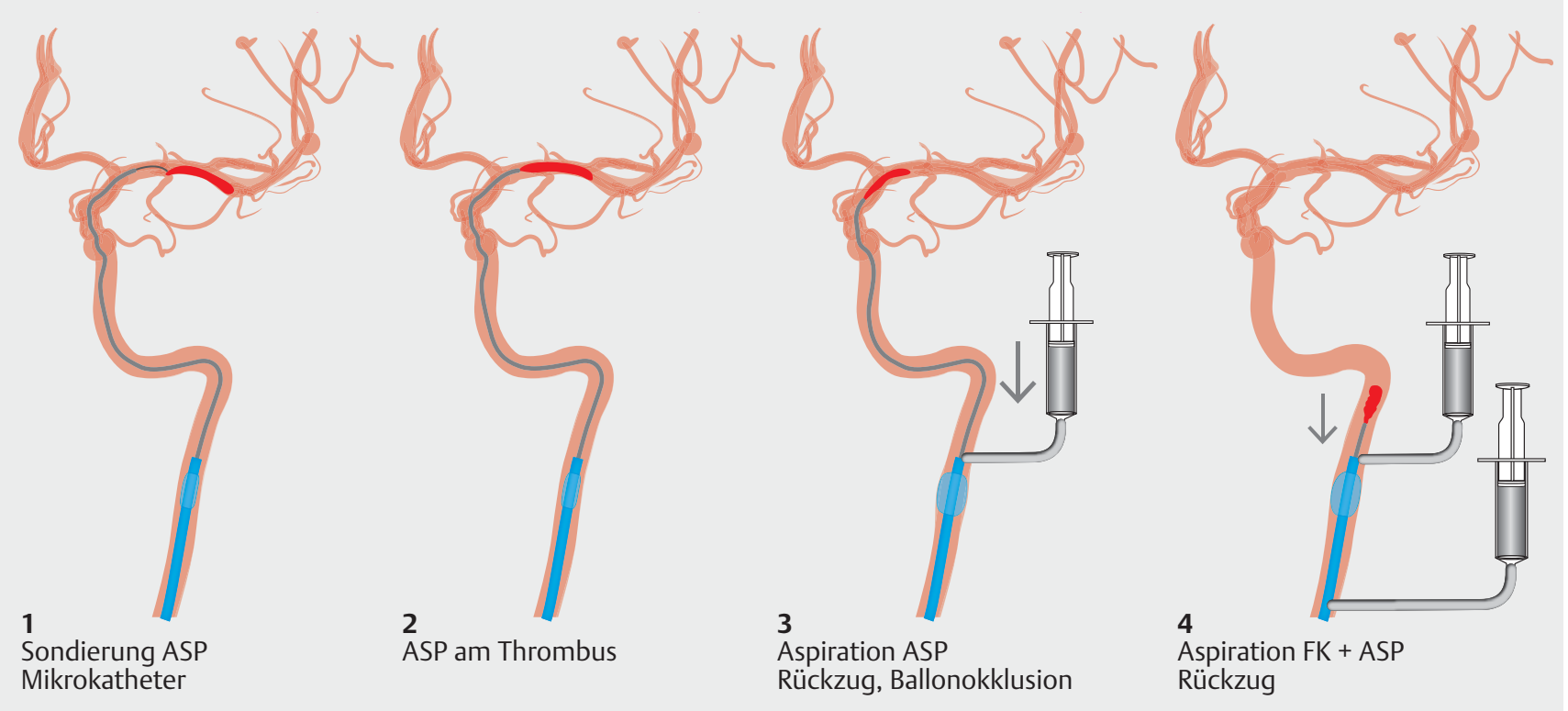

- Abb. 6 Standardisiertes Vorgehen bei der direkten Thrombusaspiration. ASP=Aspirationskatheter, FK= Führungskatheter

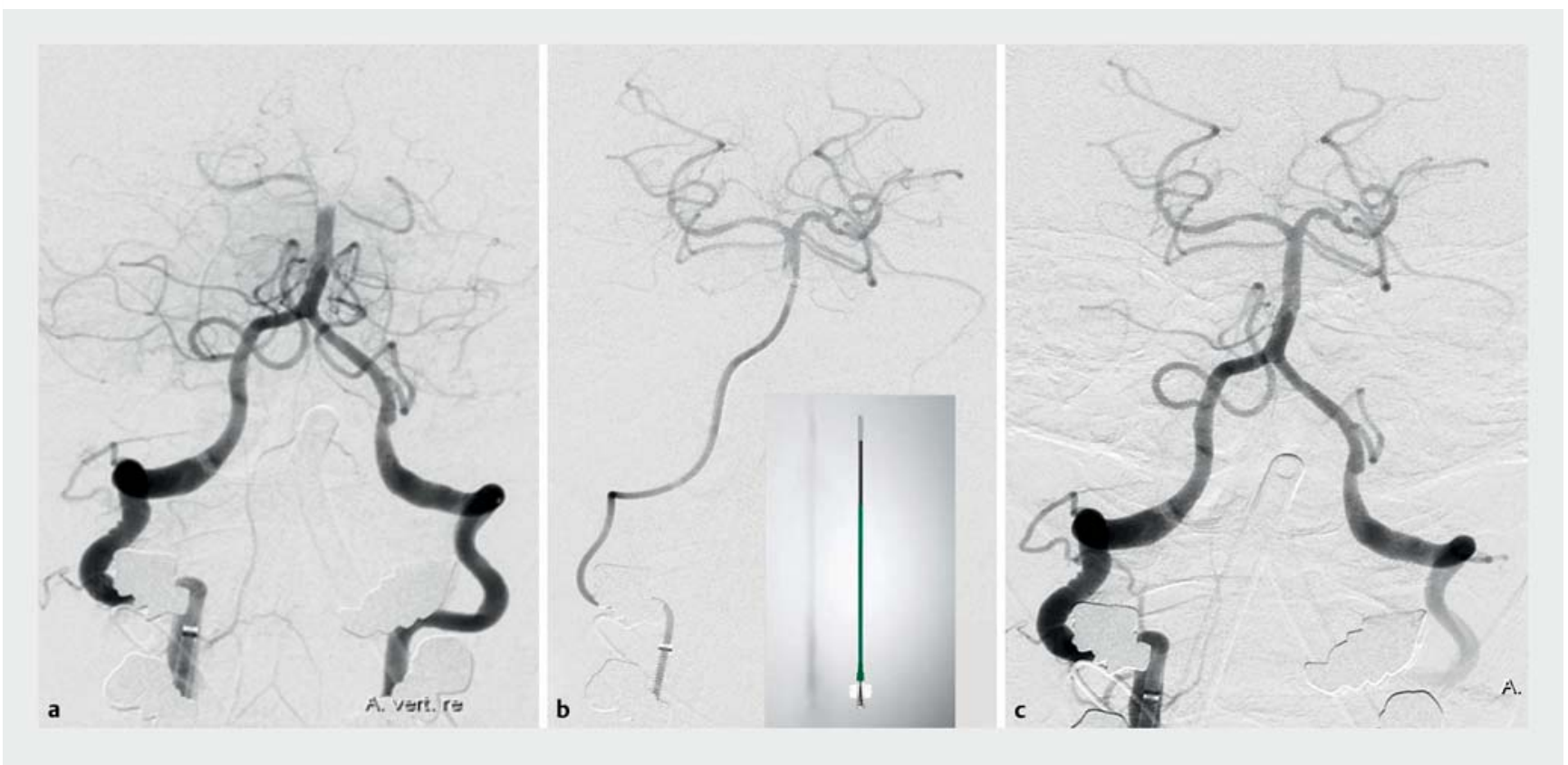

Abb.7 Fallbeispiel 2 Direkte Thrombusaspiration bei einer 69-jährigen Patientin mit Basilarisspitzenembolie. a Angiographie über eine in die V. vertebralis eingebrachte lange 6-Schleuse: Schwach umflossener Thrombus in der distalen A. basilaris. b Nach einmaliger kräftiger Aspiration mit einem 6 F-Aspirationskatheter Wiedereröffnung der Basilarisspitze. c Vollständige Rekanalisation in der Abschlusskontrolle.

Schritt 3: Nach Anschluss einer Vakuumspritze oder einer Vakuumpumpe an den in Kontakt mit dem Thrombus befindlichen Aspirationskatheter beginnt der vorsichtige Rückzug des Katheters unter kontinuierlicher Aspiration des Führungskatheters unter temporärer Ballonokklusion der zuführenden Arterie.
Schritt 4: Nach Hineinziehen in den Führungskatheter und Entfernung des Aspirationskatheters wird bei rückläufigem Führungskatheter der Ballon deflatiert. Anschließend erfolgt eine Kontrollangiographie. Bei unzureichendem Rekanalisationsergebnis kann die Aspiration wiederholt oder sekundär noch ein Stent-Retriever eingesetzt werden. 
Die direkte Thrombusaspiration hat gegenüber der Stent-Retriever-Technik den Vorteil, dass der Thrombus nicht passiert werden muss. In Studien konnte gezeigt werden, dass angiographische und klinische Erfolgssraten beider Techniken sich nicht signifikant unterscheiden. Es ist gegenwärtig noch nicht ganz klar, ob es Unterschiede bezüglich der der Häufigkeit peripherer Embolien und der Notwendigkeit, zusätzlich einen Stent-Retriever als sog. Rescue-Therapie einzusetzen, gibt [8]. In einem Thrombektomie-Zentrum sollten beide Techniken beherrscht werden. Die direkte Aspiration ist besonders für kurzstreckige, an einer Bifurkation hängende Thromben geeignet (Fallbeispiel 2). Mit großlumigen 6F-Aspirationskathetern kompatible Ballonführungskatheter stehen derzeit noch nicht für alle Systeme zur Verfügung, so dass ggf. eine lange Schleuse ohne die Möglichkeit einer temporären Ballonokklusion verwendet werden muss.

\section{Technik C: Kombination Stent-Retriever und Aspirationskatheter}

siehe $>$ Abb. 8, Fallbeispiel 3 ( $>$ Abb. 9)

Mit den beiden genannten Techniken lassen sich mit einem Thrombektomie-Manöver Rekanalisationsraten $\geq \mathrm{TICI}$ IIb von $80-90 \%$ erreichen [7,8]. Weiterentwicklungen der Standardtechniken haben zum Ziel den Anteil an erfolgreichen First-pass-Manövern zu erhöhen, um so eine schnellere Rekanalisation zu erreichen. Das zweite Ziel ist die Verminderung des Risikos der mit den beiden Standardtechniken möglichen Thrombus- fragmentierung mit Embolisation in bisher nicht betroffene Gefäßterritorien.

Ein interessanter Ansatz ist hierzu die SAVE (Stent Retriever Assisted Vacuum locked Extraction)-Technik, bei der der Thrombus mit einem Stent-Retriever unter kontinuierlicher Aspiration an die distale Öffnung des koaxial eingeführten Aspirationskatheters herangezogen wird. Der Stent-Retriever klemmt dabei den Thrombus am Aspirationskatheter ein, der unter kontinuierlicher Aspiration und zusätzlicher Aspiration des Führungskatheters zurückgezogen wird [11].

Schritt 1: Ein Koaxialsystem aus Mikroführungsdraht, Mikrokatheter und Aspirationskatheter wird in Richtung auf den Thrombus vorgeschoben. Der Thrombus wird mit dem Mikrokatheter passiert und der Stent-Retriever wird darüber freigesetzt (siehe Technik A). Der Aspirationskatheter wird an den Thrombus herangeführt. Der Mikrokatheter wird entfernt, um die Fläche für die Aspiration zu vergrößern. Der entfaltete StentRetriever bleibt dabei in Position, auch wenn man den Draht beim Rückzug des Mikrokatheters nicht festhalten kann.

Schritt 2: Unter kontinuierlicher Aspiration des Führungskatheters mit einer Vakuumpumpe oder mit eine Vakuumspritze wird der Stent-Retriever vorsichtig an den Eingang des Aspirationskatheters herangezogen und nur partiell hineingezogen. Wenn der Thrombus zwischen Katheterende und Stent-Retriever einge-

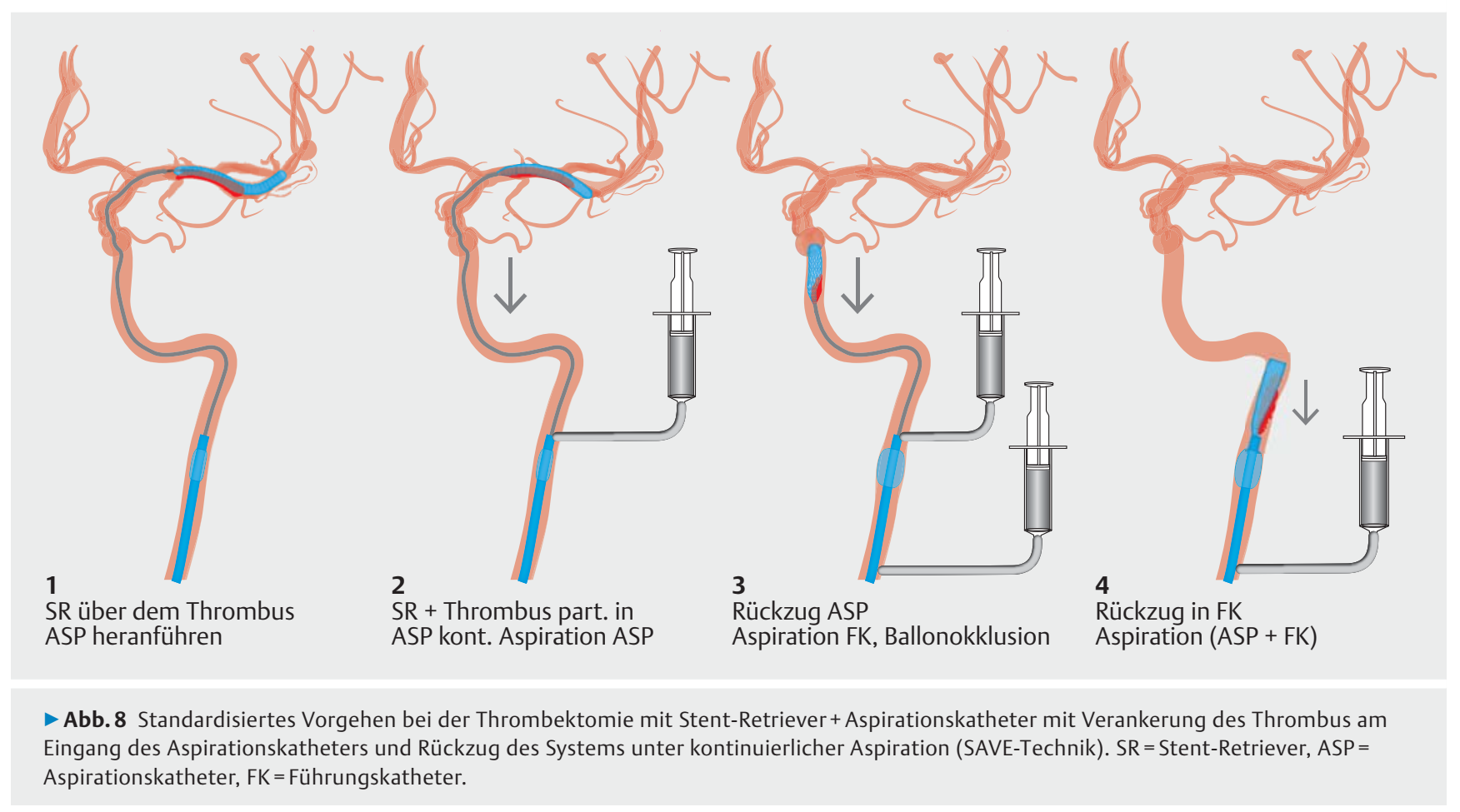




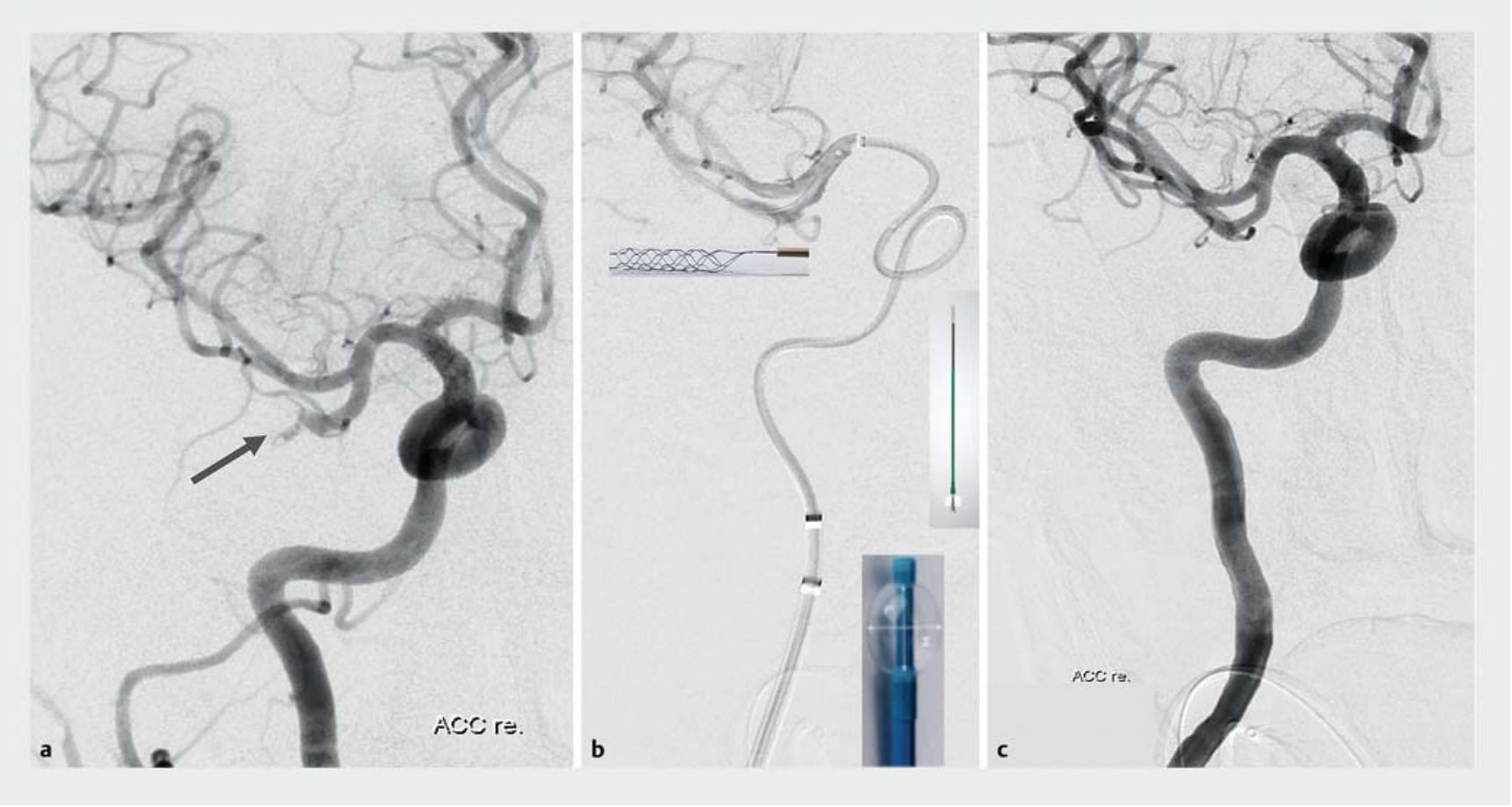

- Abb.9 Fallbeispiel 3a Embolischer Verschluss des dominanten unteren M2-Astes rechts bei einer Patientin mit Rechtshemisphärensyndrom. b Ballonführungskatheter, Aspirationskatheter und der über dem Thrombus entfaltete Stent-Retriever sind vor Ort und führen zu einer partiellen Rekanalisation. c Nach Rückzug des Stent-Retrievers und Verankerung des Thrombus am Eingang des Aspirationskatheters unter kontinuierliche Aspiration wird das Thrombektomie-System unter temporärer Ballonokklusion der ACl und zusätzlicher Aspiration des Führungskatheters geborgen. Die Abschluss-Kontrolle zeigt eine komplette Wiedereröffnung der A. cerebri media mit einem Thrombektomie-Manöver mit SAVETechnik.

klemmt ist, ist das Ostium des Aspirationskatheters verlegt und es fließt kein Blut mehr zurück.

Schritt 3: Aspirationskatheter und fixierter Stent-Retriever werden unter fortdauernder Aspiration und zusätzlicher Aspiration des Führungskatheters gemeinsam zurückgezogen. Eine temporäre Ballonokklusion des zuführenden Gefäßes ist dabei zusätzlich vorteilhaft.

Schritt 4: Wie bei den anderen Techniken auch wird die Rückläufigkeit des Führungskatheters nach vollständigem Rückzug des Thrombektomie-Systems überprüft. Nach Deflation des Ballons folgt eine angiographische Erfolgskontrolle.

Mit der SAVE-Technik lassen sich nach ersten multizentrischen Studiendaten First-pass-Rekanalisationsraten von 59\% erreichen. Im Vergleich zur ADAPT-Technik war die Rate von sehr guten TICI Ilc oder III-Rekanalisationsergebnissen höher. Nach bisherigen Ergebnissen scheinen auch die Patienten klinisch von einer möglichst raschen und vollständigen Rekanalisation zu profitieren. Im Vergleich zu anderen kombinierten Techniken mit komplettem Rückzug des Stent-Retrievers in einen Aspirations- oder Intermediärkatheter scheinen die Ergebnisse der SAVE-Technik u. a. durch eine gerin- gere Rate an distalen Embolien besser zu sein [12]. Vergleichsstudien mit höherem Evidenzniveau müssen zeigen, ob sich die komplexere, aber auch standardisiert anwendbare SAVE-Technik klinisch für die Patienten wirklich lohnt. Alternative, technisch etwas einfachere Entwicklungen versuchen einen besseren Rückhalt der Thromben durch größere Öffnungen in entsprechend modifizierten Stent-Retrievern zu erreichen [13]. Auch mit Stent-Retriever+Ballonführungskatheter lassen sich nach Registerdaten [7] ähnlich gute Ergebnisse erreichen.

\section{Angiographische Erfolgskontrolle}

Abschließende Angiogramme in 2 Ebenen sind für die standardisierte Bewertung des Rekanalisationsergebnisses nach dem TICI (Thrombolysis In Cerebral Infarction)-Score erforderlich. Abb.10 illustriert die am häufigsten verwendete Variante des modifizierten TICl-Scores [14]. Als erfolgreiche Rekanalisation gilt ein Ergebnis>2b. Gegenwärtige Entwicklungen der Thrombektomie-Techniken haben zum Ziel, eine möglichst vollständige Rekanalisation TICI Ilc oder III zu erreichen, die nochmals zu einer signifikanten Verbesserung des klinischen Behandlungsergebnisses führen kann [15]. 

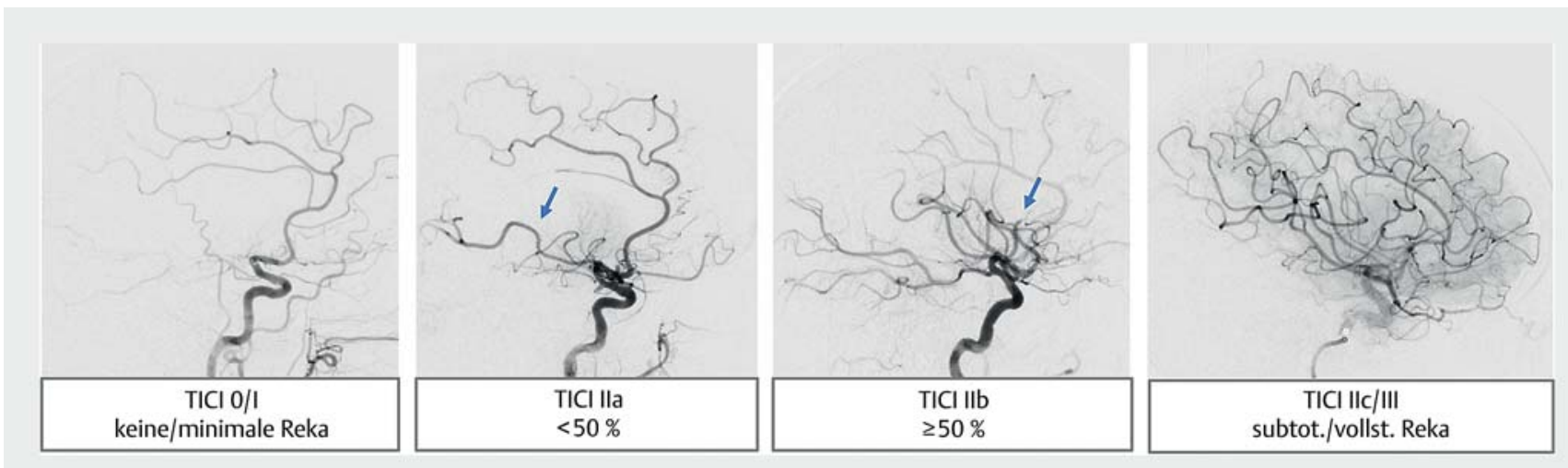

- Abb.10 Klassifikation des Rekanalisationserfolgs nach dem modifizierten TICI-Score am Beispiel von Fällen mit M1-Verschluss im lateralen Carotisangiogramm: a TICI 0 und 1: fehlende oder minimale Rekanalisation: nur A. cerebri anterior gefüllt. b TICI lla: Rekanalisation<50\% des Mediaterritoriums: nur ein Ast gefüllt (Pfeil). c TICI IIb: Rekanalisation $\geq 50 \%$ des Mediaterritoriums: peripherer Astverschluss frontal (Pfeil) d TICI IIc/III: Subtotale oder vollständige Rekanalisation: Wiedereröffnung aller Mediaäste, partiell verlagnsamte Perfusion bei TICI Ilc.

\section{Postinterventionelles Management}

Nach der Thrombektomie ist es die Aufgabe des Neurologen, die Patienten adäquat zu überwachen und weiter zu betreuen. Folgende Punkte sind dabei besonders zu berücksichtigen:

- Im Falle einer ITN sollte eine möglichst rasche Extubation, am besten noch im Angiographie-Raum angestrebt werden, um längere Beatmungen zu vermeiden und das Risiko einer Pneumonie zu verringern.

- Zur Prävention von Reperfusionsschäden mit intrazerebralen Blutungen ist nach erfolgreicher Rekanalisation ein Blutdruckmonitoring. Eine Hypertonie sollte konsequent behandelt werden. Insbesondere sollten Blutdruckspitzen > $150 \mathrm{~mm} \mathrm{Hg}$ systolisch vermieden werden. Symptomatische Blutungen kamen in den randomisierten Studien in 4,4\% der Fälle vor und waren nach Thrombektomie nicht signifikant häufiger als nach alleiniger i. v.-Thrombolyse [1].

- Eine spezielle antithrombotische Nachbehandlung ist nach Thrombektomie eines embolischen Gefäßverschlusses nicht erforderlich. Die postinterventionelle Gabe von ASS oder eine Antikoagulation bei Vorhofflimmern kann nach den neurologischen Leitlinien erfolgen.

- Geringe Subarachnoidalblutungen an der Thrombektomie-Stelle oder Kontrastmittel-Extravasate in Infarkten sind in postinterventionellen CT-Kontrollen nach $24 \mathrm{~h}$ relativ häufig nachweisbar und haben meist keine klinische Relevanz. Die Befunde sollten kontrolliert werden bevor antithrombotische Medikamente gegeben werden.

\section{Limitierungen}

Diese Anleitung bezieht sich auf die grundlegenden Schritte verschiedener Thrombektomie-Techniken und soll Verständnis für die von Neuroradiologen heute standardmäßig eingesetzten Techniken vermitteln.

Sie soll keine Anleitung für die Durchführung von Thrombektomien durch darin unerfahrene und nicht adäquat ausgebildete Ärzte darstellen.

Die DeGIR/DGNR hält dafür ein Ausbildungs- und Zertifizierungsprogramm für interventionelle Neuroradiologen und Radiologen vor, das derzeit 50 unter Aufsicht in einem anerkannten Ausbildungszentrum durchzuführende rekanalisierende Eingriffe vorsieht. Keinesfalls sollte eine Klinik mit Stroke-Unit auf die Idee kommen, allein anhand von Anleitungen, Simulatortrainings oder Modellversuchen mit der Thrombektomie zu beginnen. Katastrophale Komplikationen in Form von lebensbedrohlichen Blutungen können die Folge sein, wenn die genannten Techniken nicht adäquat eingesetzt werden.

Diese Arbeit behandelt nicht spezielle Techniken wie die Rekanalisation von Tandemläsionen mit zusätzlicher extrakranieller Stenose oder die Behandlung von Gefäßverschlüssen auf dem Boden einer intrakraniellen Stenose mit akutem Stenting. Ebenso wird nicht auf noch nicht abschließend durch Studien etablierte Indikationen wie die Rekanalisation von Patienten mit jenseits der Mediabifurkation gelegenen M2-Astverschlüssen eingegangen. 


\section{Fazit}

Das Vorgehen bei der Thrombektomie beim akuten Schlaganfall lässt sich weitgehend standardisieren. Es gibt drei grundlegende, in Thrombektomiezentren zu beherrschende Techniken: Stent-Retriever, direkte Thrombusaspiration und die Kombination aus beidem in Form der komplexeren SAVE-Technik. In jedem Zentrum sollte eine Entscheidung getroffen werden, welche Variante je nach Gefäßanatomie bevorzugt eingesetzt und standardisiert von adäquat ausgebildeten Operateuren durchgeführt wird. Eine Zusammenarbeit von Neurologen und Neuroradiologen ist beim Management von Thrombektomie-Patienten unerlässlich.

Interessenkonflikt

Mitglied der DGNR und des Berufsverbands der Neuroradiologen.

\section{Autorinnen/Autoren}

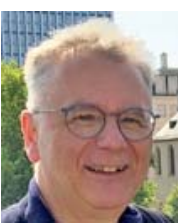

\section{Joachim Berkefeld}

Prof. Dr. med., Leitender Oberarzt am Institut für Neuroradiologie des Universitätsklinikums Frankfurt am Main. Seine klinischen und wissenschaftlichen Schwerpunkte liegen auf dem Gebiet der vaskulären und interventionellen Neuroradiologie.

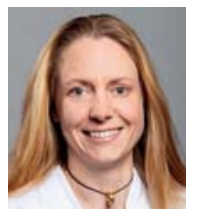

\section{Fee Keil}

Dr. med., Funktionsoberärztin am Institut für Neuroradiologie des Universitätsklinikums Frankfurt am Main. Mit interventionellem Schwerpunkt führt sie zahlreiche Thrombektomien durch und ist an der Koordination von Behandlungsstandards im Institut und anderen Einrichtungen im regionalen Schlaganfallnetzwerk beteiligt.

\section{Korrespondenzadresse}

Prof. Dr. Joachim Berkefeld

Institut für Neuroradiologie

Klinikum der Goethe-Universität

Schleusenweg 2 - 16

60528 Frankfurt am Main

E-Mail: Berkefeld@em.uni-frankfurt.de

\section{Literatur}

[1] Goyal M, Menon BK, van Zwam WH et al. Endovascular thrombectomy after large-vessel ischaemic stroke: a metaanalysis of individual patient data from five randomised trials. Lancet 2016; 387: 1723-1731
[2] Nogueira RG, Jadhav AP, Haussen DC et al. for the DAWN Trial Investigators Thrombectomy 6 to 24 Hours after Stroke with a Mismatch between Deficit and Infarct. N Engl J Med 2018; 378: 11-21

[3] Ringleb PA, Veltkamp R et al. Leitlinie Akuttherapie des ischämischen Schlaganfalls - Ergänzung 2015. Rekanalisierende Therapie AWMF 030 - 140DGN; 2015: online

[4] Pexman JH, Barber PA, Hill MD et al. Use of the Alberta Stroke Program Early CT Score (ASPECTS) for assessing CT scans in patients with acute stroke. AJNR Am J Neuroradiol 2001; 22: 1534-1542

[5] Tahtali D, Bohmann F, Rostek P et al. Setting Up a Stroke Team Algorithm and Conducting Simulation-based Training in the Emergency Department - A Practical Guide. J Vis Exp 2017; 119: doi: $10.3791 / 55138$

[6] Schönenberger S, Hendén PL, Simonsen CZ et al. Association of General Anesthesia vs Procedural Sedation With Functional Outcome Among Patients With Acute Ischemic Stroke Undergoing Thrombectomy: A Systematic Review and Meta-analysis. JAMA 2019; 322: 1283-1293

[7] Zaidat OO, Mueller-Kronast NH, Hassan AE et al. Impact of Balloon Guide Catheter Use on Clinical and Angiographic Outcomes in the STRATIS Stroke Thrombectomy Registry. Stroke 2019; 50: 697-704

[8] Turk AS, Siddiqui A, Fifi JT et al. Aspiration thrombectomy versus stent retriever thrombectomy as first-line approach for large vessel occlusion (COMPASS): a multicentre, randomised, open label, blinded outcome, non-inferiority trial. Lancet 2019; 393: 998-1008

[9] Maus V, Brehm A, Tsogkas I et al. Stent retriever placement in embolectomy: the choice of the post-bifurcational trunk influences the first-pass reperfusion result in $\mathrm{M} 1$ occlusions. J Neurointerv Surg 2019; 11: 237-240

[10] Jia B, Feng L, Liebeskind DS et al. Mechanical thrombectomy and rescue therapy for intracranial large artery occlusion with underlying atherosclerosis. J Neurointerv Surg 2018; 10: 746-750

[11] Maus V, Behme D, Kabbasch C et al. Maximizing First-Pass Complete Reperfusion with SAVE. Clin Neuroradiol 2018; 28: 327-338

[12] Brehm A, Maus V, Tsogkas I et al. Stent-retriever assisted vacuum-locked extraction (SAVE) versus a direct aspiration first pass technique (ADAPT) for acute stroke: data from the real-world. BMC Neurology 2019; 19: 65doi:10.1186/ s12883-019-1291-9

[13] Osama O, Zaidat OO, Bozorgchami H et al. Primary Results of the Multicenter ARISE II Study(Analysis of Revascularization in Ischemic Stroke With EmboTrap). Stroke 2018; 49: 1107-1115

[14] Noser EA, Shaltoni HM, Hall CE et al. Aggressive mechanical clot disruption: a safe adjunct to thrombolytic therapy in acute stroke? Stroke 2005; 36: 292-296

[15] Liebeskind DS, Bracard S, Guillemin F et al. eTICI reperfusion: defining success in endovascular stroke therapy. J Neurolntervent Surg 2019; 11: 433-438

\section{Bibliografie}

DOI https://doi.org/10.1055/a-0970-2871

Neurologie up2date 2020; 3: 221-232

(c) Georg Thieme Verlag KG Stuttgart · New York ISSN 2511-3453 